\title{
Superfluid transition temperature from the Lindemann-like criterion
}

\author{
S.M. Apenko* \\ I.E. Tamm Theory Division, P.N. Lebedev Physical Institute, Moscow, 117924, Russia
}

(November 8, 2018)

\begin{abstract}
We further analyze the recently proposed criterion, according to which a superfluid transition occurs whenever the r.m.s. displacement of a particle from its initial position in imaginary time reaches a certain fraction of the interparticle distance. The critical temperature $T_{\lambda}$ is expressed in terms of the single particle mobility. Within the Drude approximation for the mobility $T_{\lambda}$ is determined by the friction coefficient (or by the viscosity for dense liquids). To check the validity of the criterion the resulting formula is applied to non-ideal Bose gas, to liquid helium and to ${ }^{3} \mathrm{He}-{ }^{4} \mathrm{He}$ mixtures. A reasonable qualitative agreement with available experiments is obtained in all cases. In the case of the Bose gas the present approach results also in an upper bound on the peak value of $T_{\lambda} / T_{\lambda}^{0}$, where $T_{\lambda}^{0}$ is the critical temperature for the ideal gas.
\end{abstract}

PACS numbers: 67.40.-w, 05.30.Jp, 67.20.+k

\section{INTRODUCTION}

Recently considerable attention has been attracted to the long standing problem of how the critical temperature of the superfluid transition depends on density (or interaction strength) in interacting Bose systems 14. If the interaction is switched off then the temperature of the Bose-Einstein condensation is given by the well known ideal gas formula

$$
T_{\lambda}^{0}=\frac{2 \pi}{[\zeta(3 / 2)]^{2 / 3}} \frac{\hbar^{2}}{m} n^{2 / 3} \simeq 3.31 \frac{\hbar^{2}}{m} n^{2 / 3}
$$

where $n$ is the density and $m$ is the particle mass (we use units $\kappa_{B}=1$ ). This formula should be approximately valid also for an interacting system in the limit of low density. By now it is generally believed that if the density increases $T_{\lambda}$ first rise with respect to the ideal gas value, but the exact form of the correction to Eq. (11) still remains, to some extent, a point of controversy, despite the large number of recent investigations 412.

While for a weakly interacting gas there exists (at least formally) a small parameter, proportional to the scattering length, the problem looks even more difficult if the density is not small. The critical temperature should finally goes down if the density is further increased, as observed e.g. in liquid helium, primarily due to an increase in effective mass, that is believed to replace $m$ in Eq. (11) for an interacting system. Since $T_{\lambda}$ is obviously not an universal quantity (unlike critical exponents it depends strongly on microscopic details of the system) it seems reasonable simply to evaluate the transition temperature numerically for a given system, using.g. the Path Integral Monte Carlo (PIMC) method15, 16, but some analytical results, if available, would certainly lead to a better understanding. In case of dense systems with strong interaction a lattice model may provide a good starting point (see e.g. Refs. 2, 14) though the lattice introduces some additional commensuration effects not present in continuous systems.

On the other hand a simple phenomenological super fluidity criterion was proposed recently by the author 13 , which results in an analytical expression for $T_{\lambda}$ in a dense system. The approach is based on a Feynman picture of a bosonic system evolving in imaginary time, when the superfluidity is related to the appearance of permutation exchange cycles of arbitrary large length 17 . 16 . At high temperatures only the identity permutation is important in the partition function and each particle returns to its initial position in the imaginary 'time' $\beta=1 / T$. Its root mean square (r.m.s.) displacement $R$ is essentially the corresponding quantum coherence length which increases when the temperature is lowered. The superfluid transition occurs when $R$ finally reaches the value of the order of the interparticle distance $a=n^{-1 / 3}$ (at lower temperatures nontrivial permutations can be no longer ignored). This picture suggests that one can first evaluate $R(T)$ at a given temperature $T$ and then obtain an estimate for $T_{\lambda}$ e.g. from the condition $R^{2}\left(T_{\lambda}\right)=\xi a^{2}$, where $\xi$ is some numerical factor of order unity (see Sec. II for details).

This approach is similar in spirit to the well known Lindemann melting criterion, which states that a solid melts whenever the r.m.s. displacement of particles from their equilibrium positions (due to lattice vibrations) reaches a certain fraction of the nearest-neighbor distance. It is known that in many cases the ratio of these two quantities (the Lindemann ratio) is almost universal thus making the criterion quite useful for qualitative estimates (see e.g. Ref. 18). One may hope that the present approach which treats the superfluid transition as some kind of 'melting in imaginary time' may also be of use at least as a first approximation.

From the physical point of view $R$ may be regarded as a generalization of the thermal de-Broigle wavelength $\lambda_{T}=\left(2 \pi \hbar^{2} / m T\right)^{1 / 2}$, which is the coherence length for non-interacting particles at a temperature $T$. Indeed, for the ideal gas $R \sim \lambda_{T}$ and our criterion coincides with the textbook argument which states that Bose-Einstein condensation occurs whenever $\lambda_{T} \sim a$ (which yields $\left.T_{\lambda} \sim\left(\hbar^{2} / m\right) n^{2 / 3}\right)$. If the interaction is switched on, interparticle scattering will tend to reduce the coherence length, so that $R$ can be much smaller than $\lambda_{T}$. The 
physics here is essentially the same as in the decoherence due to environment 19 , since for a given particle all other particles will now act as some dissipative environment. One may also recall that the kinetic energy $K(T)$ of a particle in a quantum liquid is no longer given by $\frac{3}{2} T$ but is higher due to interaction (at low temperatures the mean kinetic energy tends to a constant value determined by a zero-point motion), so that the particle wavelength estimated as $\sim\left(\hbar^{2} / m K(T)\right)^{1 / 2}$ indeed gets smaller than $\lambda_{T}$.

If the interaction leads to the reduction of the coherence length it also reduces the superfluid transition temperature (the initial growth of $T_{\lambda}$ with interaction strength in a Bose gas has a different origint and will be discussed in Sec. III). Thus e.g. the observed decrease of $T_{\lambda}$ with pressure in helium may be understood either as a decoherence phenomenon or as a consequence of the more intensive zero-point motion in a pressurized system.

In Ref. 13 this supefluidity criterion was applied to molecular hydrogen $\mathrm{H}_{2}$ and results in $T_{\lambda} \sim 1 \mathrm{~K}$ for the temperature of the possible superfluid transition 2023. This estimate is significantly lower than the fery first one, based on the ideal gas formula (11), $6 \div 8 \mathrm{~K} 20$ (though consistent with laterestimates 21 and PIMC results for $\mathrm{H}_{2}$ cluster $\$ 24$ and film 22 ). This result was obtained, however, by assuming that the 'Lindemann' parameter $\xi$ is universal, i.e. the same as in case of liquid helium. Such an assumption may cause some doubt and its further analysis is certainly in order. The r.m.s. displacement $R$ that enters the superfluidity criterion was evaluated in Ref. 13 using the Caldeira-Leggett mode 25 which also needs some justification in our case.

In this paper we study this problems in more detail in order to better understand the accuracy and reliability of the criterion proposed. In Sec. II we review some general aspects of the theory. The general formula will be derived, which relates $R^{2}$ to the mobility of an external particle in the liquid. We will show that the CaldeiraLeggett model approach is equivalent in our case to the Drude approximation for the mobility. Within the Drude approximation the mobility is determined entirely by the collision rate $\gamma$ and it appears possible to derive a general formula for the superfluid transition temperature which is an universal function of $\hbar \gamma / T_{\lambda}^{0}$. If this ratio is much larger than unity the superfluidity is suppressed.

Next we apply the formula obtained to different superfluid systems and compare the results to available experimental data. In Sec. III a weakly interacting Bose gas is considered. Here we analyze the ratio of $T_{\lambda} / T_{\lambda}^{0}$ as a function of density and compare the result to the known PIMC calculationt. Sec. IV is deyoted to liquid helium. Contrary to the previous work 13 where $\gamma$ was related to the mean kinetic energy of a helium atom, the transition temperature is expressed here as a function of the shear viscosity in the normal state (at $T$ close to $T_{\lambda}$ ). We also check the validity of the Drude approximation and find out that for liquid helium this is quite accurate if exchange effects are neglected. Finally, in Sec. V we

extend the present approach to ${ }^{3} \mathrm{He}^{-}{ }^{4} \mathrm{He}$ mixtures. With no additional fitting it appears possible to describe qualitatively the behaviour of $T_{\lambda}$ as a function of both the total density and the concentration of ${ }^{3} \mathrm{He}$ atoms.

\section{GENERAL FORMALISM}

According to the Feynman's path integral approach 17 the partition function $Z$ for a system of $N$ interacting bosons can be represented as a sum over all possible permutations of these particles

$$
\begin{gathered}
Z=\frac{1}{N !} \sum_{P} \int \prod_{i} d \mathbf{r}_{i} \int \prod_{i} \mathcal{D} \mathbf{r}_{i}(\tau) \exp \left(-\frac{1}{\hbar} S\right), \\
S=\int_{0}^{\hbar \beta}\left[\sum_{i} \frac{m \dot{\mathbf{r}}_{i}^{2}}{2}+\sum_{i<j} V\left(\mathbf{r}_{i}-\mathbf{r}_{j}\right)\right] d \tau
\end{gathered}
$$

where $V\left(\mathbf{r}_{i}-\mathbf{r}_{j}\right)$ is the interparticle interaction potential and $\beta=1 / T$. The integration in Eq. (2) is over all paths with $\mathbf{r}_{i}(0)=\mathbf{r}_{i}, \mathbf{r}_{i}(\hbar \beta)=P \mathbf{r}_{i}$, where $P$ is some permutation of $N$ particles.

At high temperatures only the identity permutation contributes to the sum, but as $\beta$ increases, other permutations finally also become important and the system becomes superfluid. In this picture the superfluid transition is related to the appearance of long cyclic permutations (see e.g. Ref. 16 for details). This means that the critical temperature should be roughly of the same order as a temperature at which nontrivial permutations can no longer be neglected. Then we may try to estimate $T_{\lambda}$ from a criterion 13

$$
R^{2} \equiv \frac{1}{\hbar \beta} \int_{0}^{\hbar \beta}\left\langle[\mathbf{r}(\tau)-\mathbf{r}(0)]^{2}\right\rangle d \tau=\xi a^{2}
$$

where $\mathbf{r}(\tau)$ is a path of some arbitrary particle in the system of distinguishable particles and $\xi$ is a numerical factor, to be determined later. Average in this formula means the functional integration over all paths of the chosen particle $\mathbf{r}(\tau)$ with $\mathbf{r}(0)=\mathbf{r}(\beta)$

$$
\langle\ldots\rangle=\frac{1}{Z} \int \mathcal{D} \mathbf{r}(\tau)\langle\ldots\rangle \exp \left(-\frac{1}{\hbar} \int_{0}^{\hbar \beta} d \tau \frac{m \dot{\mathbf{r}}^{2}}{2}-W\{\mathbf{r}(\tau)\}\right)
$$

where the effective action $W\{\mathbf{r}(\tau)\}$ arises from the integration over other $N-1$ particles $(i=1,2 \ldots N-1)$ with $\mathbf{r}_{i}(0)=\mathbf{r}_{i}(\beta)$

$$
\begin{gathered}
\exp (-W\{\mathbf{r}\})=\int \prod_{i} \mathcal{D} \mathbf{r}_{i}(\tau) \exp \left(-\frac{1}{\hbar} S^{\prime}\left\{\mathbf{r}, \mathbf{r}_{i}\right\}\right) \\
S^{\prime}\left\{\mathbf{r}, \mathbf{r}_{i}\right\}=\int_{0}^{\hbar \beta}\left[\sum_{i} \frac{m \dot{\mathbf{r}}_{i}^{2}}{2}+\sum_{i} V\left(\mathbf{r}-\mathbf{r}_{i}\right)+\sum_{i<j} V\left(\mathbf{r}_{i}-\mathbf{r}_{j}\right)\right] d \tau
\end{gathered}
$$


The condition (3) merely states that near the transition the mean displacement (in imaginary time) of a given particle from its initial position becomes comparable to the interparticle spacing. This is similar in spirit to the well known Lindemann melting criterion. For this reason we shall some times refer to Eq. (3) as the 'Lindemannlike' criterion and also call $\xi$ the 'Lindemann ratio'. We shall assume here that $\xi$ is roughly a constant, but, certainly, possible weak dependence of $\xi$ on system parameters (e.g. on density) cannot be excluded and may be important in some cases (see e.g. Sec. III).

In the ideal gas the left hand side of Eq. (3) is essentially the square of the de Broglie thermal wavelength $\lambda_{T}^{2}=2 \pi \hbar^{2} / m T$, and our criterion looks like $\lambda_{T} \sim a$, but interactions will tend to reduce $R^{2}$ (this was observed e.g. in Ref. 26). One may think of this reduction as arising from the decoherence due to interaction with environment. Neighboring particles which scatter from the one we are looking at 'measure', in a sense, its position thus reducing its quantum uncertainty in coordinate space (compare e.g. with Ref. 19).

Estimating $R^{2}$ in a system of interacting particles is still a very complicated problem, even if exchanges are neglected. One has first to calculate somehow the effective action $W\{\mathbf{r}\}$ given by Eq. (5). However, since we are interested only in the simple average (3) we can avoid the evaluation of $W\{\mathbf{r}\}$ and relate this average to the mobility of an external particle.

If we introduce a Fourier transform of the path $\mathbf{r}(\tau)$ according to

$$
\mathbf{r}(\tau)=\sum_{n=-\infty}^{+\infty} \mathbf{r}_{n} e^{-i \omega_{n} \tau}
$$

where $\omega_{n}=2 \pi n / \hbar \beta, n=0, \pm 1, \pm 2, \ldots$ then in three dimensions the mean square displacement defined by Eq. (3) can be written as

$$
R^{2}=12 \sum_{n=1}^{+\infty}\left\langle\left|\mathbf{r}_{n}\right|^{2}\right\rangle
$$

Now let us write the expression for the mobility in the Matsubara representation $\mu_{M}\left(\omega_{n}\right)$. For this purpose we introduce an external force $\mathbf{f}(\tau)=\mathbf{f} \exp \left(-i \omega_{n} \tau\right)$ acting on a given particle and a perturbation term

$$
\delta S=-\int_{0}^{\hbar \beta} \mathbf{r}(\tau) \mathbf{f}(\tau) d \tau
$$

in the action. The force will induce the average velocity $\langle\mathbf{v}(\tau)\rangle=\mathbf{v}_{n} \exp \left(-i \omega_{n} \tau\right)$ and the mobility $\mu_{M}\left(\omega_{n}\right)$ is defined according to

$$
\mathbf{v}_{n}=\mu_{M}\left(\omega_{n}\right) \mathbf{f}
$$

Since $\langle\mathbf{v}(\tau)\rangle=i\langle\dot{\mathbf{r}}(\tau)\rangle$, to the first order in $\mathbf{f}$ we obtain

$$
\langle\mathbf{v}(\tau)\rangle=\frac{i}{\hbar} \int_{0}^{\hbar \beta}\left\langle\dot{\mathbf{r}}(\tau) \mathbf{r}\left(\tau^{\prime}\right)\right\rangle \mathbf{f}\left(\tau^{\prime}\right) d \tau^{\prime}
$$

and hence

$$
\mu_{M}\left(\omega_{n}\right)=\omega_{n} \beta\left\langle\left|\mathbf{r}_{n}\right|^{2}\right\rangle .
$$

This means that the average we are interested in can be expressed as follows

$$
R^{2}=\frac{12}{\beta} \sum_{n=1}^{+\infty} \frac{\mu_{M}\left(\omega_{n}\right)}{\omega_{n}}
$$

The Matsubara mobility is related to the mobility at real frequency $\mu(\omega)$ by the usual equation

$$
\mu_{M}\left(\omega_{n}\right)=\mu\left(i\left|\omega_{n}\right|\right)
$$

Since $\mu(\omega)$ is a response function and is analytical in the upper half-plane of the complex frequency one can easily verify that

$$
\mu\left(i \omega_{n}\right)=\frac{1}{\pi} \int_{-\infty}^{+\infty} d \omega \frac{\omega_{n} \operatorname{Re} \mu(\omega)}{\omega_{n}^{2}+\omega^{2}}
$$

for positive $\omega_{n}$. Substituting this expression in Eq. (11) and carrying out summation over $n$ we finally obtain

$$
R^{2}=\frac{6 \hbar}{\pi} \int_{0}^{+\infty} \frac{d \omega}{\omega} \operatorname{Re} \mu(\omega)\left[\operatorname{coth}\left(\frac{\hbar \omega}{2 T}\right)-\frac{2 T}{\hbar \omega}\right] .
$$

Thus if we know $\mu(\omega)$ from some theory or experiment, we can evaluate the mean square displacement (13) which enters in our superfluidity criterion.

Important asymptotics can be obtained, however, without knowing the exact expression for $\mu(\omega)$. We shall assume only that the static mobility $\mu=\mu(0)$ is finite and introduce the scattering rate $\gamma=1 /(m \mu)$. First of all, if the interaction is switched off, then $R^{2} \rightarrow \hbar^{2} \beta / 2 m$. Then the criterion (3) results in $T_{\lambda}=(1 / 2 \xi) \hbar^{2} n^{2 / 3} / m$. Since this is the ideal gas formula for the critical temperature (11) we conclude that $1 / 2 \xi \simeq 3.31$, i.e.

$$
\xi \simeq 0.15
$$

If however the interaction is so strong that $\hbar \gamma \gg T$ then the leading low temperature contribution to $R^{2}$ will be logarithmic in $\hbar \gamma / T$

$$
R^{2} \simeq \frac{6}{\pi} \frac{\hbar}{m \gamma} \ln \left(\frac{\hbar \gamma}{2 \pi T}\right)+\text { const }
$$

It is clear from (13) that this logarithmic behavior is actually independent of the form of $\mu(\omega)$ provided the static mobility is finite i.e. $\mu(0) \neq 0$, and the prefactor is determined entirely by $\mu=1 /(m \gamma)$. The second term in (15) depends on the exact form of the function $\mu(\omega)$.

It is important, that though we consider low temperatures, we always deal here with nondegenerate liquid where the mobility (and hence $\gamma$ ) is approximately independent of temperature (see Sec. III). Below the $\lambda$ point the friction of an external particle is determined by collisions with quasiparticles (phonons and rotons) and will 
tend to zero as $T \rightarrow 0$. For this reason it is more adequate to describe the system below the $\lambda$ point in terms of an effective mass $m^{*}$. Our present approach is in fact complementary to the one based on the notion of effective mass, which results in $T_{\lambda} \sim\left(\hbar^{2} / m^{*}\right) n^{2 / 3}$. The temperature dependence of $R^{2}$ in the normal system above $T_{\lambda}$ (with all exchange effects switched off) can hardly be described by a simple renormalization of the particle mass, and the incoherent diffusion may be more adequate a model for the particle motion (see also Sec. IV). Though the effective mass approach results in the apparently simple expression for $T_{\lambda}$ it is not really simpler, since there are no analytical closed expressions for $m^{*}$.

Using Eq. (15) we can obtain from Eq. (3) a very simple formula

$$
T_{\lambda}=\alpha \frac{\hbar \gamma}{2 \pi} \exp \left(-\xi \frac{\pi}{6} \frac{\hbar \gamma}{T_{0}}\right), \quad T_{0}=\frac{\hbar^{2}}{m} n^{2 / 3}
$$

where $\alpha$ is some unknown factor, dependent on the high frequency behavior of $\mu(\omega)$, and $T_{0}$ is essentially the critical temperature for the ideal gas (it differs from Eq. (11) only by a numerical factor).

Thus the temperature of the superfluid transition crucially depends on the ratio $\hbar \gamma / T_{0}$ which is a function of density and interaction strength. At $\hbar \gamma \ll T_{0}$ the ideal gas formula (11) is valid while in the opposite limit the critical temperature of the $\lambda$ transition is exponentially small due to the decoherence phenomenon. Since $\gamma$ should normally increase with density (for systems with strong repulsion between particles at small distances), the formula obtained qualitatively explains the suppression of $T_{\lambda}$ in sufficiently dense systems.

Now we introduce a simple model for $\mu(\omega)$. At high frequencies (higher than the collision rate) the mobility is the same as for a free particle, i.e. $\mu(\omega) \sim i /(m \omega)$. Then we may try to use, as a first approximation, the interpolation formula for the frequency dependent mobility in the Drude form

$$
\mu(\omega)=\frac{1}{\lambda-i m \omega}
$$

where $\lambda$ is the friction coefficient which determines the static mobility $\mu=\mu(0)=1 / \lambda$. Frequency dependence of this kind is valid for the mobility of a heavy particle, moving with friction according to the Langevin equation. Variational calculations of Ref. 27 suggest that the Drude formula may be adequate for a light particle as well. In fact this interpolation formula is reasonable when there is only one frequency scale in a problem. Strictly speaking the friction coefficient should be also frequency dependent, i.e. $\lambda=\lambda(\omega)$, but in many cases it is possible to neglect this dispersion since $\operatorname{Re} \mu(\omega)$ will be already very small when the dispersion in $\lambda$ becomes significant. Now, substituting Eq. (17) in Eq. (13) we obtain

$$
R^{2}=\frac{6}{\pi} \frac{\hbar}{m \gamma}\left[C+\psi\left(1+\frac{\hbar \gamma \beta}{2 \pi}\right)\right]
$$

where $\gamma=\lambda / m, \psi(x)$ is the digamma function and $C \simeq 0.577 \ldots$ is the Euler's constant. This very expression was used previously 13 when the motion of a particle was described by the Caldeira-Leggett model with ohmic dissipation. Note that this formula (with the suitable choice of $\gamma$ ) may be used also to describe the suppression of superfluidity by an external disordered potential (this will be discussed elsewhere).

The final result for the transition temperature in case of $\mu(\omega)$ given by Eq. (17) may be written as

$$
T_{\lambda}=\frac{\hbar \gamma}{2 \pi} F\left(\xi \frac{\pi}{6} \frac{\hbar \gamma}{T_{0}}\right), \quad \gamma=\lambda / m
$$

where the function $F(x)$ is implicitly defined by the equation

$$
x=C+\psi(1+1 / F(x)) .
$$

From Eq. (20) one can obtain $F(x) \simeq\left(6 / \pi^{2}\right) 1 / x$ at $x \rightarrow 0$ while $F(x)$ becomes exponentially small $F(x) \simeq$ $\exp (C-x)$ at $x \rightarrow \infty$. In the intermediate region $x \sim 1$ this function is well approximated by the interpolation formula $F(x) \simeq 1.6 \exp (-0.96 x) /(1-\exp (-0.96 x))$.

Unfortunately, even for such a simple choice of $\mu(\omega)$ there seems to be no opportunity to calculate the value of $\gamma$ analytically for a dense liquid with a strong interaction between particles. For this reason one has to use experimental results as well as approximations with some adjustable parameters. One can also try to use the fluctuation-dissipation theorem for the velocity fluctuations and write the following expression for the kinetic energy

$$
K(T)=\frac{3}{2} m \int_{0}^{\infty} \frac{d \omega}{\pi} \hbar \omega \operatorname{Re} \mu(\omega) \operatorname{coth}\left(\frac{\hbar \omega}{2 T}\right)
$$

If we put $T=0$ then

$$
K=\frac{3}{2} m \int_{0}^{\infty} d \omega \hbar \omega \operatorname{Re} \mu(\omega)
$$

where $K=K(0)$. This integral is known to diverge logarithmically for the Drude mobility at high frequencies. If we assume that for true $\mu(\omega)$ there is only one characteristic frequency scale $\gamma$ then obviously

$$
K \sim m \hbar \gamma^{2} / \lambda \sim \hbar \gamma
$$

This approximation was used in the previous papert3, and it has some advantage, because the kinetic energy $K$ (essentially the zero-point energy) can be easily evaluated for various systems 28 . In what follows, however, we shall try to make a more direct estimate of the damping parameter $\gamma$.

\section{WEAKLY INTERACTING BOSE GAS}

Before we turn to dense liquids consider first a nonideal Bose gas. In the gas the transition temperature 
is close to Eq. (11) and we are interested in corrections to $T_{\lambda}^{0}$ at non-zero density. To estimate these corrections consider a classical gas of hard spheres of diameter $d$. Then the scattering rate is given by

$$
\gamma \sim v / l \sim \pi n d^{2} v
$$

where $l \sim 1 /\left(n \pi d^{2}\right)$ is the mean free path and $v \sim$ $(T / m)^{1 / 2}$ is the mean velocity. Since $T \sim T_{\lambda}^{0} \sim$ $\hbar^{2} n^{2 / 3} / m$

$$
\gamma \sim \frac{\hbar}{m} d^{2} n^{4 / 3}
$$

If we introduce a small parameter $z=\left(n d^{3}\right)^{1 / 3}$ then we see that the ratio $\hbar \gamma / T_{\lambda}^{0} \sim z^{2}$ is small at low densities and one can expand the formula (18) for $R^{2}$ in $\hbar \gamma / T$. Retaining only the first correction in $\gamma$ we find from Eq. (3)

$$
T_{\lambda} \simeq \frac{1}{2 \xi} \frac{\hbar^{2}}{m} n^{2 / 3}\left(1-\frac{3 \zeta(3)}{\pi^{3}} \frac{\hbar \gamma}{T_{\lambda}^{0}}\right)
$$

The correction due to decoherence is negative and leads to the suppression of $T_{\lambda}$. It is generally accepted, however, that the first correction to Eq. (11) should be positive 9,12 . The only way to account for this increase of $T_{\lambda}$ in the present approach is to assume that the parameter $\xi$ also slightly depends on density and gets smaller as the density is increased i.e.

$$
\xi=\xi(n) \simeq \xi_{0}(1-\delta)
$$

where $\xi_{0} \simeq 0.15$ and the small density dependent correction $\delta$ will be specified later. The physical reason for such a dependence is the same as in Ref. 6]: particles in a more dense system tend to be more homogeneously distributed through the whole volume, hence it is easier for a particle to find a neighbor for exchange. Then we can write

$$
\frac{T_{\lambda}}{T_{\lambda}^{0}} \simeq 1+\delta-\frac{3 \zeta(3)}{\pi^{3}} \frac{\hbar \gamma}{T_{\lambda}^{0}}
$$

Now we need some more accurate estimate of $\gamma$. For a nondegenerate system of hard spheres one can evaluate the friction coefficient $\lambda=m \gamma$ from the Bolzmann equation and in the low density limit

$$
\lambda=\frac{32}{3} n d^{2}(\pi m T)^{1 / 2}
$$

(see e.g. Ref. 29). The additional factor of 4 is introduced here because in our case, contrary to the pure classical one, the wavelength of the particle is larger then $d$ and the $s$-wave scattering cross section is known to be $4 \pi d^{2}$ rather than $\pi d^{2}$ as for a classical sphere.

Hence near the transition, when $T \simeq T_{\lambda}^{0}$

$$
\hbar \gamma \simeq \frac{32 \sqrt{2} \pi}{3[\zeta(3 / 2)]^{1 / 3}} \frac{\hbar^{2}}{m} d^{2} n^{4 / 3}
$$

Most of recent calculations suggest, that at low density the first positizecfrrection in Eq. (27) should be of the first order in $d 46$, 60 in this limit we can take $\delta \sim z$. At higher density the Lindemann ratio is likely to reach some finite limit close to $\xi \simeq 0.12 .13$ Then we may try a simple interpolation formula

$$
\delta=\frac{a_{1} z}{1+a_{2} z}
$$

Then the final expression for the transition temperature can be written as

$$
T_{\lambda} / T_{\lambda}^{0} \simeq 1+\frac{a_{1} z}{1+a_{2} z}-a_{3} z^{2}, \quad z=\left(n d^{3}\right)^{1 / 3}
$$

where $a_{3} \simeq 1.2$ from Eqs.(27) and (29), while $a_{1}$ and $a_{2}$ should be taken from some microscopic theory or experiment. The dependence of $T_{\lambda} / T_{\lambda}^{0}$ on density given by Eq. (31) is shown in Fig. 1 for $c_{4} \simeq 2.3$ as obtained e.g. in recent Monte Carlo study 8 and for $a_{2} \simeq 9.9$. This value of $a_{2}$ was fixed by the requirement that at $z \simeq 0.61$ (which approximately corresponds to helium density, when $\left.n d^{3} \simeq 0.23\right) \xi$ should be close to 012 i.e. $\delta \simeq 0.2$. The squares represent the PIMC datal for a quantum system of hard spheres. Though we have taken rather large initial slope $a_{1} \simeq 2.3$, which is almost an order of magnitude larger than reported in Ref. 7, the resulting curve does not deviates much from the PIMC data (the largest deviation is less than ten percent) and reveals a qualitatively similar behaviour.

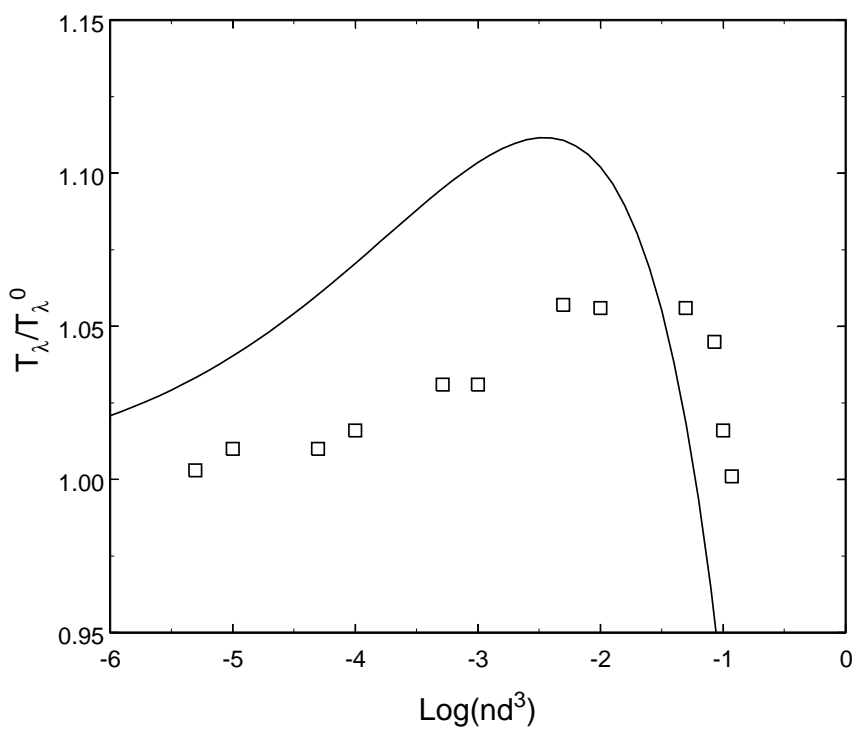

FIG. 1. Critical temperature $T_{\lambda}$ of an interacting Bose gas versus density from Eq. 31 with $a_{1}=2.3, a_{2}=9.9$ and $a_{3}=1.2\left(T_{\lambda}^{0}\right.$ is the critical temperature of the ideal gas, $d$ is the hard-sphere diameter). For comparison, PIMC simulation results are also included.

What is perhaps more interesting, explaining the initial rise of $T_{\lambda}$ with density by a change in the Lindemann ratio $\xi$ places an upper bound on the peak value of $T_{\lambda} / T_{\lambda}^{0}$. 
Indeed, if the parameter $\xi$ changes from $\xi_{0} \simeq 0.15$ to something like $\xi \sim 0.12$ at high densities then the maximum value of $\delta$ in Eq. (27) is 0.2 and hence $T_{\lambda} / T_{\lambda}^{0}$ should not exceed 1.2. This result is consistent with the PIMC calculations but is incompatible with recent experiment in ${ }^{4} \mathrm{He}-\mathrm{Vycor}$ system 10 where the peak value of $T_{\lambda} / T_{\lambda}^{0}$ was found to be almost 3.5 times larger. This disagreement seems to be not yet explained and one may suspect that we do not fully understand all possible effects of a disordered porous substrate in Vycor samples on $T_{\lambda}$.

\section{LIQUID HELIUM}

Let us see first what an elementary kinetic approach can suggest for $\mu(\omega)$. It is known from experiment 30 that above the superfluid transition the momentum distribution of helium atoms is approximately of a Gaussian type with the mean kinetic energy $K \sim 15 \mathrm{~K}$. If we used a Boltzman equation for a classical gas of hard spheres of diameter $d$ at effective temperature $T^{*}=\frac{2}{3} K$ then in the relaxation time approximation we obviously would obtain the frequency dependent mobility in the Drude form (17) with the inverse relaxation time $\gamma \sim v / l \sim \pi n d^{2} v$ as in Eq. (23) where now $v \sim(K / m)^{1 / 2}$. At the same time one has

$$
\lambda \sim m \gamma \sim \pi n d^{2} m v
$$

for the friction coefficient and

$$
\eta \sim m v n l \sim(1 / \pi) m v / d^{2}
$$

for shear viscosity. From these formulas we get

$$
\lambda \sim \pi^{2}\left(n d^{3}\right) \eta d
$$

Extrapolating this result to a dense system with $n d^{3} \sim 1$ we see that the friction coefficient in a liquid is in fact proportional to the viscosity. Note that the estimate (32) is quite reasonable for liquid helium above the $\lambda$ point 31 . Indeed, the measured viscosity above $T_{\lambda}$ is almost independent of temperature and is equal to $\eta \simeq 3.5 \cdot 10^{-5}$ poise (at s.v.p. and at $T=3.2 \mathrm{~K}$ ) while the more accurate version of Eq. (32) $\eta \simeq 0.18\left(m T^{*}\right)^{1 / 2} / d^{2}$ gives $\eta \simeq 3.6 \cdot 10^{-5}$ poise at $d \simeq 2.2 \AA$.

Practically the same result as Eq. (33) arises also from the hydrodynamic approach. For a particle of a large radius $\mathcal{R} \gg a$ in a liquid the friction coefficient $\lambda$ from the Stokes' formula is $6 \pi \eta \mathcal{R}$. It is clear, however, that for a particle of the liquid, which is not large as compared to other particles, this formula should be modified. Long ago it was argued (see e.g. Ref. 32 and references therein) that it this case one should rather take $\lambda=4 \pi \eta \mathcal{R}$, as for an empty bubble in a liquid. One may suppose in this connection that a particle in a liquid is surrounded by a 'correlation hole', so that one may view the particle as associated with a 'bubble' of radius $\mathcal{R} \sim n^{-1 / 3}$. Then we have an estimate

$$
\lambda=4 \pi \eta b n^{-1 / 3}
$$

where we have put $\mathcal{R}=b n^{-1 / 3}$ and $b$ is some numerical factor of order unity. Certainly, this estimate makes sense only above the $\lambda$ transition and for sufficiently dense liquid. When $n d^{3} \sim 1$, estimates (33) and (34) lead to essentially the same results. In what follows we shall use Eq. (34) since this will result in a slightly more transparent final formulas.

Now, making use of Eq. (34) we can rewrite the general formula (19) for the superfluid transition temperature in the form

$$
T_{\lambda}=A \frac{\hbar^{2}}{m} n^{2 / 3} \frac{\eta}{\eta_{0}} F\left(B \frac{\eta}{\eta_{0}}\right), \quad \eta_{0}=\hbar n
$$

where the function $F(x)$ is defined by Eq.(20) and

$$
A=2 b, \quad B=\frac{2}{3} \pi^{2} b \xi
$$

We see now that the critical temperature in this approach is determined by the shear viscosity $\eta$, and $T_{\lambda}$ will be smaller than the ideal gas value if the viscosity is considerably larger than $\eta_{0}=\hbar n$, which may be called a 'quantum' of viscosity. For typical densities of liquid helium one has $\eta_{0} \simeq 2 \cdot 10^{-5}$ poise, while the viscosity above the superfluid transition is $\eta \simeq 3.5 \cdot 10^{-5}$ poise. Thus in the case of helium $\eta$ is only slightly larger than $\eta_{0}$, and hence the temperature of the $\lambda$ transition should be close to that of the ideal gas, as it is experimentally. In sufficiently viscous Bose liquids the critical temperature of the possible superfluid transition should be suppressed and they will crystallize on cooling.

The viscosity in helium slightly above $T_{\lambda}$ is almost independent of temperature and increases with density approximately by a factor of two when the pressure is raised from s.v.p. to $24 \mathrm{~atm}$. We take experimental data for the pressure dependent viscosity at $T=3.2 \mathrm{~K}$ from Ref. 33 and turn it into $\eta(n)$ dependence using the known equation of state at the same temperature. The density dependence of the ratio $\eta / \eta_{0}$ is approximated then by the second order polynomial

$$
\eta / \eta_{0} \simeq \sum_{l=0}^{2} c_{l}\left(n^{*}\right)^{l}
$$

where $n^{*}=n r_{0}^{3}\left(r_{0} \simeq 2.556 \AA\right)$ is the reduced density and the coefficients are $c_{0} \simeq 2.965, c_{1} \simeq-15.701$ and $c_{2} \simeq 34.027$. Then we fit Eq. (35) to the experimental data for $T_{\lambda}$ in helium (actually to the Kierstead empirical equation for the $\lambda$ line 34 ) and obtain $A \simeq 1.39$ and $B \simeq$ 0.58 , which correspond to quite reasonable values

$$
b \simeq 0.7, \quad \xi \simeq 0.13
$$

The resulting curve for the transition temperature is shown in Fig. 2 (solid line). We also extrapolate this curve to lower densities, corresponding to a metastable 
liquid, though we do not expect our hydrodynamic formula (34) to be valid at $n \sim 0.2 \div 0.3$. At low densities the friction coefficient should be smaller than Eq. (34) (see e.g. Eq. (33)) and hence the true value of $T_{\lambda}$ should be higher. Also on this figure the condensation temperature for the ideal gas is shown (dashed line) along with the experimental data for liquid helium (squares) and recent PIMC results for metastable helium at negative pressure 35 (circles). Note that the points at negative pressure were not used in the fit.

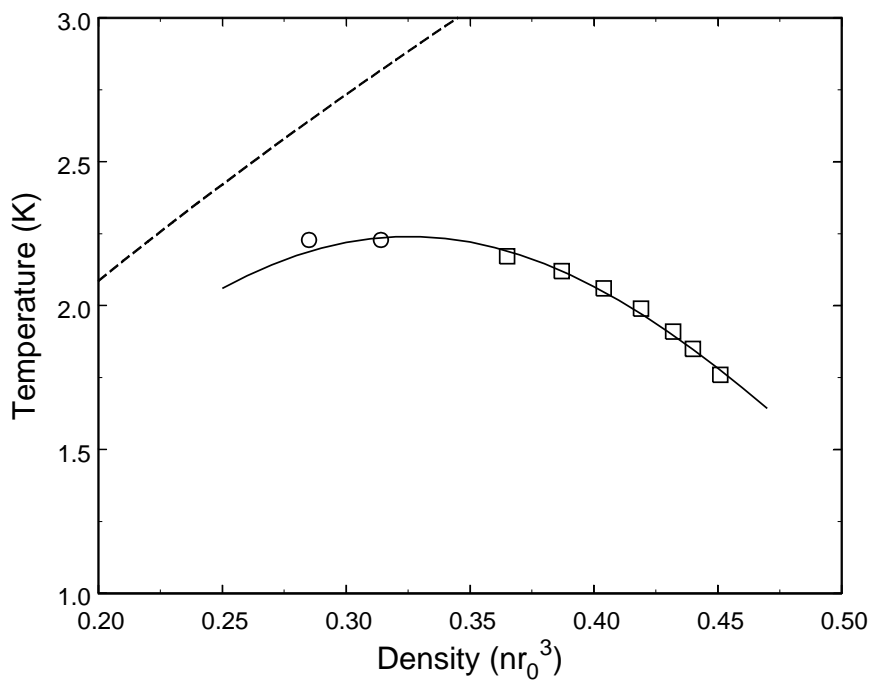

FIG. 2. Temperature of the superfluid transition versus the reduced density $n^{*}=n r_{0}^{3}\left(r_{0}=2.556 \AA\right)$. Solid line is the theory (35) at $A=1.39, B=0.58$, squares denotes experimental data for helium, dashed line corresponds to the ideal gaf. Recent simulation results for helium at negative pressure 35 are also included (circles).

The value of the Lindemann ratio $\xi$ from Eq. (38) is quite close to that of the ideal gas $\xi_{0} \simeq 0.15$ and also to $\xi \simeq 0.12$ obtained in Ref. 13 by taking $\hbar \gamma \sim K$. If we now evaluate the value of the damping parameter $\gamma=\lambda / m$ then at $b \simeq 0.7$ we have from Eq. (34) $\hbar \gamma \simeq 13 \mathrm{~K}$, which is indeed close to the zero point kinetic energy $K \simeq 15$ $\mathrm{K}$.

In the case of helium it appears possible to check also the validity of the Drude approximation (17) which results in Eq. (18) for the mean square displacement in imaginary time. For this purpose let us consider a more general quantity

$$
R^{2}(\tau)=\left\langle[\mathbf{r}(\tau)-\mathbf{r}(0)]^{2}\right\rangle
$$

The $R^{2}$ discussed above is just the average of $R^{2}(\tau)$ over imaginary time. Proceeding as in Sec. II and taking the mobility in the Drude form (17) we obtain

$$
R^{2}(\tau)=\frac{24}{m \beta} \sum_{n=1}^{+\infty} \frac{1}{\omega_{n}^{2}+\gamma \omega_{n}} \sin ^{2}\left(\frac{\omega_{n} \tau}{2}\right)
$$

Note that for a free particle one would have

$$
R^{2}(\tau)=(3 / m \beta) \tau(\hbar \beta-\tau)
$$

At small $\tau$ we can replace the sum in Eq. (39) by the integral to obtain $R^{2}(\tau) \simeq(3 \hbar / m) \tau$ which is independent of $\gamma$ since on a very short time scale the particle moves without collisions. In the general case the sum can be calculated numerically and the result for $\beta=2$ $\mathrm{K}^{-1}$ and $\hbar \gamma \simeq 13 \mathrm{~K}$ (as obtained from the fitting of $T_{\lambda}$ ) is shown by the solid line in Fig. 3. Also the PIMC calculation results are shown as presented in Ref. 16, where the same quantity was evaluated for bosons (triangles) and for distinguishable particles (squares).

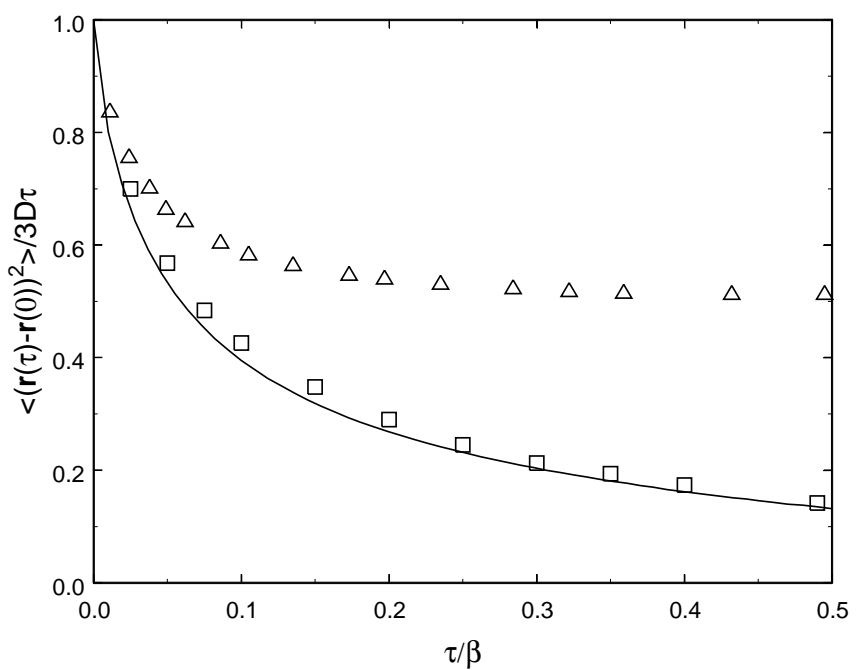

FIG. 3. The diffusion of ${ }^{4} \mathrm{He}$ atoms as a function of imaginary time $\tau$ for $T=0.5 \mathrm{~K}(D=\hbar / m$ and $\beta=1 / T)$. The solid line results from $\mathrm{Eq}-39$ at $\hbar \gamma=13 K$. For comparison, PIMC simulation results 16 are also included, both for bosons (triangles) and distinguishable particles (squares).

Is is clear from Fig. 3 that the Drude approximation well account for the depression of $R^{2}(\tau)$ due to the interaction in the nondegenerate system. Since by our definition $R^{2}$ should be calculated for the system with all exchange effects neglected (see Sec. II) we conclude that the Drude approximation (and hence the CaldeiraLeggett model) may be quite sufficient for our superfluidity criterion.

\section{V. ${ }^{3} \mathrm{HE}-{ }^{4} \mathrm{HE}$ MIXTURES}

To check the universality of the Lindemann-like criterion it is necessary to consider some other superfluid systems. A natural choice to start with is the case of ${ }^{3} \mathrm{He}-{ }^{4} \mathrm{He}$ mixtures. One may try to repeat calculations from the previous section and express $T_{\lambda}$ in terms of viscosity. Unfortunately there seems to be no such viscosity measurements for a wide range of pressures as that of Goodwin 33 for pure ${ }^{4} \mathrm{He}$. Though there exists a recent investigation 36 of $\eta$ in mixtures it deals only with pressures near the saturated vapor pressure. Above $T_{\lambda}$ (at 
$T \sim 3 \mathrm{~K})$ the viscosity at s.v.p. is almost independent of temperature and gets smaller if the concentration $x$ of ${ }^{3} \mathrm{He}$ atoms increases.

At small $x$ this effect may be attributed simply to the decrease of the total density. Indeed, adding ${ }^{3} \mathrm{He}$ atoms to liquid ${ }^{4} \mathrm{He}$ leads to some reduction of the mean density (due to lighter mass of ${ }^{3} \mathrm{He}$ atoms they have larger zeropoint energy and are harder to localize) and hence to a lower viscosity. But at a fixed density the local environment of a given ${ }^{4} \mathrm{He}$ atom remains practically the same as for $x=0$ since interactions between atoms do not depend on the isotope mass. Hence the viscosity and the mean square displacement $R^{2}$ in dilute mixtures (small $x)$ are likely to be the same as in pure ${ }^{4} \mathrm{He}$ at the same density.

However, it is the mean distance between Bose particles that enters in the r.h.s. of the criterion (3). This distance is now larger by the factor $(1-x)^{-1 / 3}$ due to the presence of additional ${ }^{3} \mathrm{He}$ atoms which do not participate in superfluidity, so the criterion should read

$$
R^{2}=\xi(1-x)^{-2 / 3} a^{2}
$$

where $a=n^{-1 / 3}$ is determined by the total mean density.

Now assuming that at a fixed density $R^{2}$ is indeed the same as in pure ${ }^{4} \mathrm{He}$, and proceeding as in the previous section we obtain

$$
T_{\lambda}=A \frac{\hbar^{2}}{m} n^{2 / 3} \frac{\eta}{\eta_{0}} F\left(\frac{1}{(1-x)^{2 / 3}} B \frac{\eta}{\eta_{0}}\right), \quad \eta_{0}=\hbar n
$$

where $\eta / \eta_{0}$ is again given by Eq. (37). The universality hypothesis means that $\xi$ is independent of $x$, so that we may consider $A$ and $B$ to be defined by Eqs. (36) and (38). Then the only dependence of the superfluid transition temperature on $x$ is through the explicit factor of $(1-x)^{-2 / 3}$ in Eq. (42).

To check the validity of the assumptions made we are to compare the formula (42) with experimental data. Here we make use of experiments 37 where $T_{\lambda}$ was measured in ${ }^{3} \mathrm{He}-{ }^{4} \mathrm{He}$ mixtures under pressure at several values of $x$. We transform these results into $T_{\lambda}(n)$ dependence using equations of state for different $x$ at $T=1.5$ $\mathrm{K}$, which hardly can introduce any significant error. These data are shown by squares in Fig. 4 for $x=0.089$ and $x=0.228$ along with the pure ${ }^{4} \mathrm{He}$ results. Solid curves represent $T_{\lambda}$ as obtained from Eq. (42). Note that only the $x=0$ curve was fitted to experiment.

We see that qualitatively the formula (42) correctly describes the dependence of the transition temperature both on density and on $x$, though for larger $x$ 's the theoretical curves tend to lie lower than the experimental points. It is difficult to decide unambiguously whether this deviation indicate possible weak dependence of $\xi$ on $x$ or simply the approximations made in evaluating $R^{2}$ are too crude. In any case the estimate (42) makes sense only for small $x$.

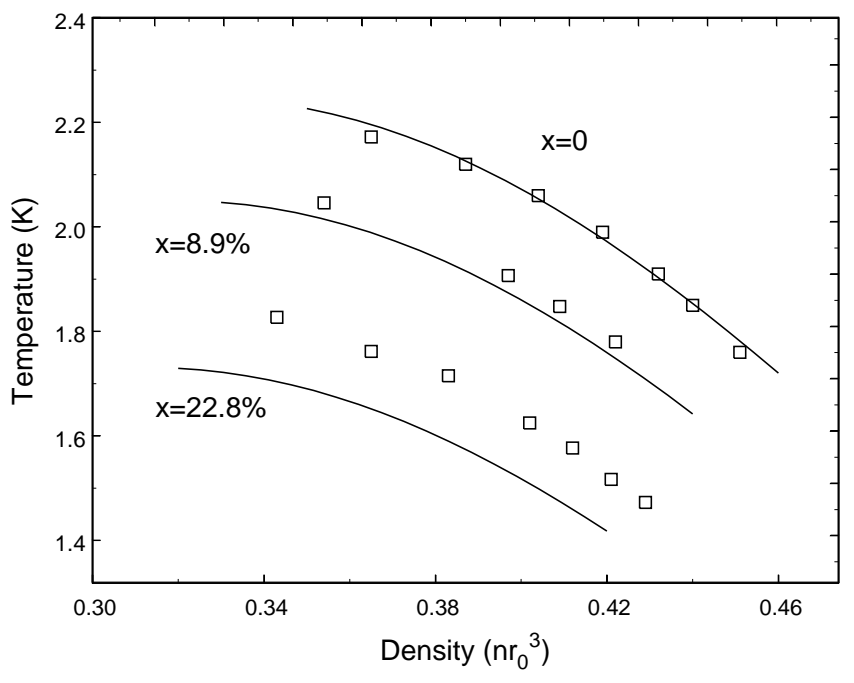

FIG. 4. Critical temperature of ${ }^{3} \mathrm{He}-{ }^{4} \mathrm{He}$ mixtures versus the reduced density $n^{*}=n r_{0}^{3}\left(r_{0}=2.556 \AA\right)$ for ${ }^{3} \mathrm{He}$ concentrations $x=0,8.9 \%$ and $22.8 \%$. Solid line is the theory (42) with $A$ and $B$ the same as for pure ${ }^{4} \mathrm{He}$, while squares represent experimental data. Only the $x=0$ curve was fitted to experiment.

\section{CONCLUSIONS}

In this paper the Lindemann-like superfluidity criterion proposed earlier 13 was applied to several systems where the superfluid transition is known to occur, namely to a weakly non-ideal Bose gas, liquid helium and ${ }^{3} \mathrm{He}-$ ${ }^{4} \mathrm{He}$ mixtures. The mean square displacement $R^{2}$ of a particle in imaginary time which enters in the criterion, and hence $T_{\lambda}$ were related here to the zero frequency friction coefficient (or to the shear viscosity in case of liquids). We found that in all cases the results obtained do qualitatively reproduce the observed behaviour of the transition temperature $T_{\lambda}$, as obtained from experiment (including numerical modeling).

As far as the quantitative accuracy of the approach is concerned the situation is still not fully understood. It is clear, however, that one can hardly expect it to be very accurate. Indeed, the criterion proposed may be of real interest only if the critical temperature differs significantly from the ideal gas value (in the opposite case the estimate (1) is quite sufficient). But it is obvious e.g. from Eq. (16), valid at $\hbar \gamma / T_{0} \gg 1$, that the result in this case is rather sensitive to the exact values of the damping parameter $\gamma$ and the phenomenological parameter $\xi$ since they both stand in the exponential. These parameters are in general not known exactly and even a small variation in $\gamma$ or $\xi$ may significantly influence the resulting estimate for $T_{\lambda}$.

It seems therefore that the best use of the formulas obtained may be the analysis of how the transition temperature, which e.g. is known for a given system, may change if some parameters of the system are slightly modified. In this case one can fix all adjustable parameters 
by fitting to the known data and then analyze their deviat ons for a modified system. A simple example of how this can be done was given in Sec. V where ${ }^{3} \mathrm{He}-{ }^{4} \mathrm{He}$ mixtures were discussed. We have first fixed all free parameters comparing the theory to the pure ${ }^{4} \mathrm{He}$ data and then take account of the nonzero concentration of ${ }^{3} \mathrm{He}$ atoms.

The criterion proposed leads also to an unexpected prediction for the weakly interacting Bose gas. In this case the initial increase of $T_{\lambda}$ with interaction strength may be attributed to the change of the Lindemann parameter $\xi$ and this results in an upper bound on the peak value of $T_{\lambda} / T_{\lambda}^{0}$. Indeed, in the limit of zero density $\xi \simeq 0.15$ and at helium densities we have an estimate $\xi \sim 0.12 \div 0.13$ (see Ref. 13 and Sec. IV) so that the change in $\xi$ and hence the maximum value of $T_{\lambda} / T_{\lambda}^{0}$ could not be large. In Sec. III we obtain a rough stimate $T_{\lambda} / T_{\lambda}^{0}<1.2$ consistent with the PIMC results]. Possible numerical evaluation of $\xi$ in a dense system can make this inequality more accurate.

Numerical estimates of $\xi$ would be desirable also from the general point of view. This is practically the only way to make a final decision concerning the validity of the approach presented here. One has to calculate $R^{2}$ along the $\lambda$ line and then check whether $\xi=R^{2} / a^{2}$ can be regarded as a constant (at least in some density range) and to what extent is $\xi$ universal in systems with e.g. different interaction potentials.

I am grateful to P.I. Arseyev and V.V. Losyakov for usefull discussions and to D.M. Ceperley for stimulating correspondence concerning the Bose gas. This work was supported in part by the Russian Foundation for the Fundamental Research (Project No 00-15-96698).

* Electronic address: apenko@lpi.ru

${ }^{1}$ T.D. Lee and C.N. Yang, Phys. Rev. 112, 1419 (1957).

2 T. Matsubara and H. Matsuda, Progr. Theor. Phys. 16, 569 (1956).

${ }^{3}$ T. Toyoda, Ann. Phys. (NY) 141, 154 (1982).

${ }^{4}$ H.T.C. Stoof, Phys. Rev. A 45, 8398 (1992); M. Bijlsma and H.T.C. Stoof, Phys. Rev. A 54, 5085 (1996).

${ }^{5}$ K. Huang, cond-mat/9904027.

${ }^{6}$ G. Baym, J.-P. Blaizot, and J. Zinn-Justin, condmat/9907241; G. Baym, J.-P. Blaizot, M. Hol, F. Laloë, and D. Vautherin, Phys. Rev. Lett. 83, 1703 (1999).

${ }^{7}$ P. Grüter, D.M. Ceperley, and F. Laloë, Phys. Rev. Lett. 79, 3549 (1997).

${ }^{8}$ M. Holzmann and W. Krauth, Phys. Rev. Lett. 83, 2687 (1999).

${ }^{9}$ M. Holzmann, P. Grüter, and F. Laloë, Eur. Phys. J. B 10, 739 (1999).

10 J.D. Reppy, B.C. Crooker, B. Hebral, A.D. Corwin, J. He, and G.M. Zassenhaus, Phys. Rev. Lett. 84, 2060 (2000).
${ }^{11} \mathrm{M}$. Wilkens, F. Illuminati, and M. Krämer, condmat/0001422.

${ }^{12}$ A.M.J. Schakel, cond-mat/0004142.

13 S.M. Apenko, Phys. Rev. B 60, 3052 (1999).

${ }^{14}$ K. Ziegler, cond-mat/0001074.

15 D.M. Ceperley and E.L. Pollock, Phys. Rev. Lett. 56, 351 (1986).

${ }^{16}$ D.M. Ceperley, Rev. Mod. Phys. 67, 279 (1995).

${ }^{17}$ R.P. Feynman, Phys. Rev. 91, 1291 (1953); 91, 1301 (1953).

18 J.P. Hansen, B. Jancovici, and D. Schiff, Phys. Rev. Lett. 29, 991 (1972).

${ }^{19}$ C. Kiefer and E. Joos, in Quantum Future, ed. P. Blanchard and A. Jadczyk (Springer, Berlin, 1998), quantph/9803052; H.D. Zeh, in Decoherence: Theoretical, Experimental and Conceptual Problems, ed. by P.Blanchard, D. Giulini, E. Joos, C. Kiefer, and I.-O. Stamatescu (Springer, 1999), quant-ph/9905004.

${ }^{20}$ V.L. Ginzburg and A.A. Sobyanin, Sov. Phys. JETP Lett. 15, 242 (1972).

${ }^{21}$ H.J. Maris, G.M. Seidel, and T.E. Huber, J. Low Temp. Phys. 51, 471 (1983); H.J. Maris, G.M. Seidel, and F.I.B. Williams, Phys. Rev. B 36, 6799 (1987).

22 M.C. Gordillo and D.M. Ceperley, Phys. Rev. Lett. 79, 3010 (1997).

${ }^{23}$ V.S. Vorob'ev and S.P. Malyshenko, Pis'ma ZhETF 71, 59 (2000).

${ }^{24}$ P. Sindzingre, D.M. Ceperley, and M.L. Klein, Phys. Rev. Lett. 67, 1871 (1990).

${ }^{25}$ A.O. Caldeira and A.J. Leggett, Phys. Rev. Lett. 46 , 211 (1981); Ann. Phys. (NY) 149, 374 (1983).

${ }^{26}$ C.L. Cleveland, Uzi Landman, and R.N. Barnett, Phys. Rev. B 39, 117 (1989).

${ }^{27}$ A. Rosch, Ph.D. thesis, Univ. Karlsruhe (Shaker Verlag, Aachen, 1997).

28 A.M. Dyugaev, J. Low Temp. Phys. 78, 79 (1990).

${ }^{29}$ C.A. Croxton, Liquid State Physics (Cambridge University Press, 1974), Ch. 6.

${ }^{30}$ A.D.B. Woods and V.F. Sears, Phys. Rev. Lett. 39, 415 (1977).

${ }^{31}$ R.D. Taylor and J.G. Dash, Phys. Rev. 106, 398 (1957).

${ }^{32}$ R.O. Herzog and H.C. Kudar, Zs. Phys., 80, 217 (1933).

33 J.M. Goodwin, Physica 76, 177 (1974).

${ }^{34}$ H.A. Kierstead, Phys. Rev. 162, 153 (1967).

${ }^{35}$ G.H. Bauer, D.M. Ceperley, and N. Goldenfeld, condmat/9909169.

${ }^{36}$ S.Wang, C.Howald, and H.Meyer, J. Low Temp. Phys. 79, 151 (1990).

${ }^{37}$ C. Le Pair, K.W. Taconis, R. De Bruyn Ouboter, P. Das, and E. De Jong, Physica 31, 764 (1965). 